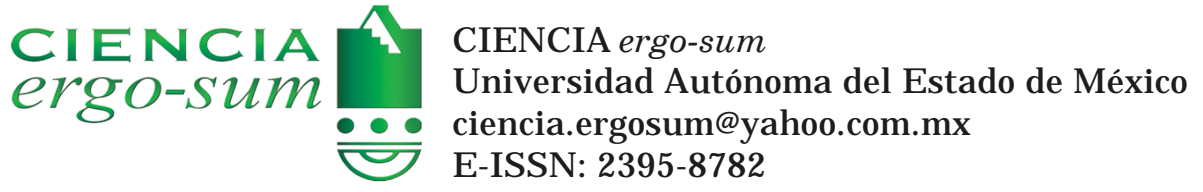

\title{
Mujeres del noreste de México que participan en proyectos productivos agropecuarios: análisis psicosocial de sus procesos de empoderamiento
}

\footnotetext{
Cazares-Palacios, Itzia María

Mujeres del noreste de México que participan en proyectos productivos agropecuarios: análisis psicosocial de sus procesos de empoderamiento

CIENCIA ergo-sum, vol. 27, núm. 1, marzo-junio 2020|e69

Universidad Autónoma del Estado de México, México

Esta obra está bajo una Licencia Creative Commons Atribución-NoComercial-SinDerivar 4.0 Internacional.
}

Cazares-Palacios, I. M. (2020). Mujeres del noreste de México que participan en proyectos productivos agropecuarios: análisis psicosocial de sus procesos de empoderamiento. CIE NCIA ergo-sum, 27(1). https://doi.org/10.30878/ces.v27n1a1 


\title{
Mujeres del noreste de México que participan en proyectos productivos agropecuarios: análisis psicosocial de sus procesos de empoderamiento
}

\author{
Women from northeastern Mexico who participate in agricultural productive projects: A psychosocial analysis of their \\ empowerment processes \\ Itzia Maria Cazares-Palacios \\ Universidad Autónoma del Estado de Hidalgo, México \\ itzia_cazares@uaeh.edu.mx \\ Recepción: 27 de junio de 2018 \\ Aprobación: 21 de marzo de 2019
}

\begin{abstract}
Resumen
Se tiene como propósito conocer de qué forma la participación de mujeres de la región sureste de Coahuila en proyectos productivos contribuye o no a sus procesos de empoderamiento psicosociales en las dimensiones individual, colectiva y de las relaciones cercanas. Tomando como base el Modelo Tridimensional de Empoderamiento de Jo Rowlands (1997), se analizaron entrevistas semiestructuradas y entrevistas grupales. Los resultados muestran que las participantes mejoran la percepción sobre sus capacidades para realizar cambios, fortalecen su confianza y autonomía; sin embargo, no se observaron transformaciones de género al interior de la unidad doméstica. En términos colectivos, se observó que el chisme es un elemento que dificulta la organización. Se discuten algunas implicaciones metodológicas para estudios futuros.
\end{abstract}

Palabras ClaVE: empoderamiento, género, rural, proyectos.

\begin{abstract}
The purpose of this research was to find how the participation of women from the southeast region of Coahuila in productive projects contributes or not, to their processes of empowerment in the individual, collective and close relationships dimensions. Based on Rowlands' Empowerment Model, semi-structure interviews and focus groups were analyzed. The results show that the participants improve their own perception about their abilities to make changes, strengthen their confidence and autonomy. However, no gender transformations were observed inside the domestic unit. In collective terms, it was observed that gossip is an element that hinders the organization. Some methodological implications for future research are discussed.
\end{abstract}

KEYWORDS: empowerment, gender, rural, projects.

\section{INTRODUCCIÓN}

De acuerdo con el CONEVAL (Consejo Nacional de Evaluación de la Política Social de Desarrollo), en el 2016, según el Informe de Pobreza en México, $46.3 \%$ de la población mexicana se encontraba en situación de pobreza ${ }^{[1]}$ y $7.6 \%$ en pobreza extrema; en este sentido, según el lugar de residencia, la población que habita en zonas rurales presenta un mayor índice de pobreza (58.3\%) en comparación con la que se encuentra en zonas urbanas (39.2\%) (CONEVAL, 2017).

El CONEVAL (2015) señala que la gran mayoría de la población rural en condiciones de pobreza tiene un potencial económico y productivo no aprovechado lo suficientemente, razón por la cual, con la intención de reducir las condiciones de pobreza de este sector, se diseñan y aplican distintos programas federales con salidas productivas que le permitan aumentar su productividad y generar ingresos económicos de forma sostenible.

Las mujeres rurales, de acuerdo con la Organización de las Naciones Unidas para la Alimentación y la Agricultura (FAO, por sus siglas en inglés, 2006), realizan funciones importantes en el desarrollo económico y social de 
sus comunidades: actividades de reproducción y producción alimentaria para el autoconsumo de sus familias y producción en el mercado, participación en la gestión de los recursos naturales como la tierra y el agua, así como en la preservación de la biodiversidad. Sin embargo, señala el organismo, las estadísticas oficiales subestiman el valor de su trabajo y su contribución general a la riqueza nacional.

Por ejemplo, de acuerdo con el Banco Mundial (2011), la participación de las mujeres en actividades remuneradas y el control de los ingresos del hogar procedentes de lo que ellas mismas ganan o transfieren, además de tener efectos positivos en el crecimiento económico de un país, modifica los patrones de gasto en formas que benefician a sus hijas e hijos en términos nutricionales, educativos, así como en el bienestar general de la familia.

En México, a partir de datos analizados en los referentes de la Cuenta Satélite, ${ }^{[2]}$ Alberti et al. (2014) concluyen que el trabajo doméstico realizado por las mujeres rurales crea plusvalía y riqueza económica, desarrolla la economía del cuidado para atender las necesidades básicas familiares y asegura el equilibrio social.

La FAO (2006) sugiere como una medida importante incrementar el acceso y el control de las mujeres sobre la tierra y otros recursos productivos a partir de programas o políticas destinados a mejorar la seguridad alimentaria o combatir la pobreza.

En relación con lo expuesto, el empoderamiento económico ${ }^{[3]}$ de las mujeres rurales o indígenas es un tema que ha formado parte de los acuerdos mundiales que el gobierno mexicano ha suscrito para dar cumplimiento a los compromisos planteados en la Cuarta Conferencia Mundial de la Mujer en Beijing, a los Objetivos del Milenio 2015 y en la actualidad a los 17 Objetivos de Desarrollo Sostenible de la Agenda 2030 de la Organización de las Naciones Unidas, con el propósito de reducir su estatus de pobreza, el de sus familias y el de sus comunidades, reducir la desigualdad, incrementar su acceso a los recursos y a una plena ciudadanía, además de luchar contra el cambio climático.

Una de las acciones para alcanzar ese empoderamiento económico es a través de la incorporación de las mujeres rurales en los procesos de desarrollo, así como de su participación en programas de financiamiento de microcréditos para desarrollar proyectos productivos.

Es muy importante facilitar condiciones que potencien el empoderamiento de las mujeres rurales y mejoren sus condiciones de vida materiales y sociales; sin embargo, los mecanismos para lograrlo, así como los resultados son discutidos desde hace bastante tiempo según estudios y fuentes diversas.

Por un lado, desde hace más de dos décadas, se señaló que muchos de esos programas estaban basados en estereotipos tradicionales del trabajo de las mujeres y se restringían a considerar su papel reproductivo social (bienestar), carecían de un análisis de las estructuras y relaciones de poder entre los géneros (equidad), se centraban en las necesidades básicas de las mujeres (antipobreza) y las consideraban como un recurso humano de desarrollo (eficiencia) (Mingo, 1996; Moser, 1991; León, 2003).

Por otro lado, se ha discutido la ambigüedad con la que es utilizada la noción de empoderamiento, la tergiversación de su sentido original enraizado en las luchas feministas y su ubicación en una concepción voluntarista; el diseño de estrategias tendientes al empoderamiento de las mujeres que soslayan el contexto histórico y presente, no las consideran como sujetas de conocimiento y desatienden sus necesidades e intereses prácticos y estratégicos, así como la carencia de un análisis interseccional que permita identificar qué sistema de desigualdad social tiene mayor peso en las condiciones de las mujeres rurales, por mencionar algunos (Batliwala, 1997; León, 1997, 2003; Rowlands, 1997; Young, 1997).

De igual forma, algunos estudios señalan que la participación de las mujeres en estos proyectos no tiene un impacto en sus procesos de empoderamiento individual, colectivo y en sus relaciones cercanas (Barreda y Gandara, 2007; Mingo, 1996; Riaño y Okali, 2008), aun cuando tomen decisiones que beneficien a sus hijas e hijos (Chablé et al., 2007); otros indican que, no obstante que estas acciones no modifican ni disminuyen la situación de pobreza de las mujeres rurales y sus familias, sí tienen un impacto en los procesos psicológicos en la dimensión individual y, en algunos casos, generan cambios en las relaciones de género al interior de las unidades domésticas (Aguilar Pinto et al., 2017; Ghebre-Medhin y Restrepo, 2002; González y Rosas, 2013; Hidalgo, 2002; Martínez et al., 2005; Pérez et al., 2008; Tuñón Pablos, 2011). 
Rowlands (1997) señala que incluso cuando las mujeres pueden empoderarse a sí mismas al obtener algún control en diversos aspectos en su cotidianidad, esto es en una dimensión individual, centrarse en ésta no es suficiente. Por su parte, Kabeer (1997) y Batliwala (1997) indican que cuando el empoderamiento de las mujeres se opera y analiza en el nivel individual se pasan por alto aspectos que tienen que ver con la forma como el poder a través de las lógicas de género es institucionalizado en diversos campos e instituciones sociales que refuerzan y perpetúan la dominación masculina tales como las leyes y derechos de propiedad de la tierra, las dificultades que enfrentan para acceder y controlar los recursos económicos, el control ejercido por parte de sus parejas o familias políticas que limitan sus posibilidades de acción o las aíslan y las estructuras organizacionales desiguales dentro de los ejidos que limitan su representación política y la toma de decisiones públicas, por ejemplo, en la gestión del agua (Deere y León, 2002; Gutiérrez et al., 2013; López y Rojas, 2017; Mora et al., 2016; Vázquez-Luna et al., 2013).

En los procesos de empoderamiento de las mujeres, la noción de poder se plantea bajo un esquema democrático y de poder compartido, lo cual es el resultado de tres tipos de poderes: poder desde dentro, poder con y poder para (Rowlands, 1997), que en suma se traducen en poderes positivos o vitales para eliminar situaciones de opresión en las vidas de las mujeres y en su entorno (Lagarde, 2012).

Por lo descrito, este trabajo se sitúa en el Modelo Tridimensional de Empoderamiento de Rowlands (1997: 224), el cual trata de "un conjunto de procesos psicológicos que, cuando se desarrollan, capacitan al individuo o al grupo para actuar e interactuar con su entorno de tal forma que incrementa su acceso al poder y su uso en varias formas".

Basada en su experiencia de trabajo con mujeres hondureñas, esta autora argumenta que un enfoque que pretenda facilitar procesos de empoderamiento requiere la consideración de dimensiones individuales, colectivas y de las relaciones cercanas, así como un análisis en cada una de los factores que los impulsan o inhiben.

Rowlands (1997) señala en la dimensión personal que la autoconfianza, la autoestima, el sentido generador de cambios, el sentido de "ser" en un contexto amplio y la dignidad son los procesos psicológicos que facilitarían un incremento en las habilidades de las mujeres para formular y expresar ideas y opiniones o de participar e influir en nuevos espacios, así como en sus habilidades para aprender, analizar y actuar, de organizar el tiempo personal e interactuar fuera del hogar, para obtener/controlar recursos y desarrollar el sentimiento de que todo es posible.

En la dimensión colectiva, el núcleo del empoderamiento se funda en la identidad del grupo, en el sentido de la capacidad colectiva para producir cambios, en la autoorganización, gestión y en la dignidad. Los indicadores de empoderamiento en esta esfera son las habilidades de negociación con otras organizaciones, incluyendo las oficiales, las habilidades para organizar las propias necesidades, de responder colectivamente a eventos externos del grupo, para reunirse e iniciar redes de organización, de generar recursos externos y el reconocimiento de otras personas y habilidades.

En la dimensión de las relaciones cercanas, la autora ubica como núcleo del empoderamiento el desarrollo de habilidades de negociación, comunicación, obtención de apoyo, defensa de los derechos y dignidad para influir en la naturaleza de la relación y la toma de decisiones al interior de ella, recursos que permitan un incremento del control sobre las circunstancias personales (ingreso, fecundidad, salidas, uso del tiempo, posibilidad de salir a reuniones), del respeto personal y de otros y en la capacidad de tomar decisiones propias.

Rowlands (1997) señala que en estos procesos se torna necesario identificar los aspectos del contexto o de la organización que impulsan o inhiben los procesos de empoderamiento y también cómo interactúan. Respecto a los factores que inhiben el empoderamiento, en términos generales, en las tres dimensiones identifica machismo, pobreza, problemas de salud, comunidad sin cohesión, opresión internalizada desde fuera y políticas locales inestables. Asimismo, el acceso a recursos productivos como la tierra y el agua, al igual que el derecho de la propiedad de la tierra, serían condiciones materiales que facilitarían el empoderamiento de las mujeres, así como también el resultado de este proceso (Braidotti, 2004; Deere y León, 2002).

Referente a los factores impulsores, se distingue el tiempo para sí mismas, compartir problemas, obtener apoyo, formar parte de un grupo y participar en sus actividades, terminar el aislamiento, identificar necesidades propias, 
desarrollo de liderazgo, autonomía, redes de trabajo con otras organizaciones, concepto de los "derechos de las mujeres", percepción crítica de la desigualdad y entrenamiento para el manejo de conflictos, por mencionar algunas.

Con base en lo expuesto, este artículo tuvo como objetivo conocer de qué forma la participación de mujeres rurales de la región sureste del estado de Coahuila en proyectos productivos contribuye, o no, a su empoderamiento en las tres dimensiones descritas. La intención es abonar desde un enfoque psicosocial a la discusión y aportar información que sea útil para el diseño, análisis y la práctica dirigida a potenciar el empoderamiento de las mujeres rurales, así como al desarrollo social y económico de sus familias y comunidades.

\section{MÉTodo}

Desde un enfoque crítico e interpretativo (Creswell, 1998), y a través de las experiencias de las participantes, la investigación se llevó a cabo a través de una aproximación cualitativa con un alcance exploratorio y descriptivo. Esta estrategia permitió distinguir las características que definen a las mujeres y a los hombres en un contexto social, económico y cultural determinado, las relaciones de género, así como los recursos y la capacidad de acción con la que cuentan las mujeres de este estudio para enfrentar las dificultades institucionales y cotidianas (Lagarde, 1996).

\section{Participantes}

Los estudios cualitativos buscan personas que presenten características teóricas relevantes, por lo cual se realizó un muestreo no probabilístico selectivo o intencionado (Patton, 1990).

Participaron 31 mujeres con una media de edad de 45 años, en su mayoría casadas, con niveles de instrucción de primaria y secundaria y con un promedio de 4 hijos. Sus giros de producción consistieron en huertos de traspatio para autoconsumo, crianza de cerdos, gallinas y nopal, productos de higiene personal herbolarios y a base de leche de cabra, frutas en conserva, licores y mermeladas, venta de nuez y tortillas de nopal.

En relación con las características de los financiamientos que recibieron las mujeres para la realización de los proyectos productivos, se distinguieron montos bajos que iban de entre $\$ 3500.00$ y $\$ 8000.00$ pesos y, en algunos casos, en calidad de créditos.

\section{TÉCNiCas de REColección de datos}

Se llevó a cabo la técnica de observación participante, la cual fue documentada a través de diarios de campo, y se realizaron entrevistas semiestructuradas y entrevistas grupales. De acuerdo con Kvale (2007), la entrevista es un medio que permite comprender lo que las personas hablan acerca de su mundo vivido y abre el intercambio entre quienes participan con el fin de construir un conocimiento compartido. Los ejes de análisis se ubicaron en las dimensiones e indicadores del empoderamiento individual, colectivo y de las relaciones cercanas expuestas.

\section{Procedimiento}

El trabajo de campo se realizó en la región sureste del estado de Coahuila, en distintas localidades de Saltillo (Tanque del Cerro, La Encantada, San José de la Joya), de General Cepeda (Narigua y El Clavel) y en diversas colonias de los municipios de Arteaga y Saltillo.

Para comenzar, las participantes se contactaron mediante instancias de la administración pública federal y estatal que otorgan financiamientos para desarrollar proyectos productivos; sin embargo, debido a la demora en la respuesta del apoyo solicitado para que nos refirieran con la población objetivo, se acudió también a instituciones educativas del nivel superior, a una asociación civil y al mercado comunitario del municipio de Arteaga. 
A cada una de las participantes se le presentaron los objetivos de la investigación y se solicitó su consentimiento para audiograbar las entrevistas, las cuales se realizaron en sus casas y en espacios comunitarios, y en horarios y condiciones que les resultaron más cómodas.

Las entrevistas individuales tuvieron una duración promedio de 60 minutos y las entrevistas grupales entre 90 y 120 minutos. Posteriormente, se transcribieron con el procesador de textos Word, se condensaron, codificaron y categorizaron (Kvale, 2007) con base en las dimensiones e indicadores señalados. Al finalizar, se les realizó un análisis cualitativo de contenido.

La confiabilidad de esta investigación está relacionada con la autenticidad de la información, y para lograrlo se utilizaron audiograbadoras. Por otra parte, siguiendo a Bartra (2012), las investigaciones no están libres de valores ni de influencias sociales, de ahí que se asume una posición feminista en este estudio que abona en el análisis de las desigualdades de género.

\section{Resultados}

En términos generales se identificó que los elementos que facilitaron o fortalecieron el empoderamiento de las mujeres en las tres dimensiones fueron el conocimiento y la relación que tienen con la naturaleza, las habilidades de solución de problemas vinculados con la subsistencia y sostenimiento familiar y la realización de actividades que les generan ingresos económicos.

Por el contrario, se observó que los elementos que restringían su empoderamiento fueron la distribución inequitativa en la unidad familiar del trabajo doméstico, labores de crianza y de cuidado, el chisme, condiciones privativas -escasez de agua- para actividades de autoconsumo y de producción individual y colectiva, y desconocimiento por parte de los operadores de los programas sociales sobre los contextos específicos, así como de las condiciones materiales, sociales y culturales en que se operan.

\section{Dimensión INDIVIDUAL DEL EMPODERAMIENTO}

De principio, un elemento vinculado con la condición de ruralidad fue el conocimiento y la relación que sostienen las participantes con su entorno natural, detonantes para obtener alguna utilidad económica que contribuyera al sostenimiento de sus vidas familiares.

Se trata de saber cómo aprovechar los recursos de tu comunidad, el campo te da trabajo y te da alimentación, tal vez no te da dinero, pero no te deja caer, siempre hay algo de qué aprovechar[...] Aprendes a disfrutar lo que estás haciendo, sembrar, cultivar, verlo crecer. Me gusta observar y practicar lo que aprendo, mi inquietud son las plantas, los animales, las piedras[...] Aprendí por mí misma a conocer para qué servían las plantas, para hacer mi negocio (Sonia, productos de higiene personal herbolarios). ${ }^{[4]}$

Dios nos ha dado la naturaleza para de cierta forma explotarla sin depredarla. Comencé vendiendo piedras, una vez pasando un arroyo de ahí de Arteaga comencé a observar, entonces, me llamó la atención las piedras de colores y yo dije voy a iniciar un negocio nuevo que nadie lo tenga y comencé a juntar piedras[...] De lo mismo de las piedras encontré los recursos para el otro negocio y así es como me encuentro vendiendo ahorita medicina tradicional (Rosa, productos herbolarios medicinales).

Estas citas muestran cómo las participantes con autoconfianza, con un reconocimiento positivo y un sentido de dignidad respecto de lo que les brinda el campo, aprovechan y capitalizan los conocimientos que tienen sobre él. Es importante mencionar que estos conocimientos y habilidades fueron las razones que motivaron a las participantes a buscar apoyos económicos; en el caso de la primera participante para otorgarle un valor agregado al transformar la materia prima (las plantas en productos herbolarios de higiene personal y con propiedades curativas) y en el caso de la segunda como un medio para potenciar el surgimiento de otros proyectos. 
Es significativo mostrar las habilidades que las participantes ponen en marcha frente a las condiciones privativas que enfrentan para la subsistencia familiar.

De las chivas obtengo leche, queso, nomás que, pues, tienen que saberle su tiempo, porque cuando ya se seca la chiva ya no da leche, y esas cosas una las debe saber para poder consumirla y venderla (Norma, productos a base de leche de cabra).

Aquí nosotros, cuando no hay tortillas, las tenemos que hacer, cuando no hay frijoles, los tenemos que cocer, aquí no hay tiendas que digamos ahí venden frijoles, ahí venden tortillas, y así aprovechamos también para vender (Clara, huertos de traspatio).

El conocimiento que tienen las participantes sobre los animales de su entorno y agricultura facilita el despliegue de habilidades para el autoconsumo y producción. Este punto pone en cuestión que exista una separación de las esferas privadas y públicas al evidenciar que las mujeres transitan en ambos espacios realizando actividades no remuneradas - producción de alimentos para el autoconsumo-y remuneradas -elaboración de alimentos para la venta-. No obstante, esto representa un exceso de trabajo para las mujeres sin un reconocimiento explícito sobre el valor y contribución que tiene en sus vidas familiares.

A mí me gustan mucho los animales y cuando mi esposo tiene ganas de ayudarme, me ayuda, y cuando no, yo ya sé lo que se va a hacer (Laura, huertos de traspatio).

No teníamos agua, esa era otra y nosotras teníamos que ir, pues yo creo que como a medio kilómetro, teníamos que ir a acarrear en carretillas el agua y la llevábamos hasta allá para lavarles y darles de comer, pero era muy pesado (Alicia, producción de nopal y crianza de cerdos).

Es sustantivo todo el trabajo que realizan las mujeres para el sostenimiento de sus vidas, así como lo excesivo que se convierte cuando carecen de recursos básicos naturales para la satisfacción de sus necesidades humanas, tal como el agua.

El agua es un derecho y un bien básico en la vida humana, pero al ser las mujeres rurales quienes realizan gran parte de las actividades domésticas y de reproducción de la vida social la privación de este recurso natural las impacta de forma distinta en comparación con los hombres, como se muestra en las citas. De ahí que Ruíz (2012) señale que un factor clave para que las mujeres tengan control sobre los recursos naturales es el reconocimiento y legitimidad social de sus necesidades y prioridades como campesinas, pero también, como lo sugieren Gutiérrez et al. (2013), es fundamental su participación y toma de decisiones en la gestión del agua en la esfera pública con el fin de impactar en la transformación de las relaciones de género.

Referente a las capacidades psicosociales que las participantes fortalecieron como resultado del acceso y control de recursos económicos, se identificaron la disposición de su tiempo, la habilidad de interactuar fuera del hogar, el reconocimiento de amplitud de acción que otorga la autonomía económica y el sentimiento de que las cosas son posibles.

Yo no le digo a mi viejo, yo no tengo, yo me salgo y regreso a la hora que sea (Luz, huertos de traspatio).

No debemos de depender de nadie[...] la mujer debe de proveer, pero también de proveerse a ella[...] si tienes un trabajo te sientes más libre. Yo pienso que es lo mejor, que la mujer trabaje y que su dinero lo traiga para ella, que lo gastamos siempre en los hijos[...] que es para lo que trabajas al final de cuentas[...] pero si traes lo tuyo te da más seguridad (Angélica, producción de frutas en conserva y mermelada).

Se aprecia que el acceso y control de los ingresos económicos generados por las propias participantes les permite reconocer la amplitud de acción que puede darles; sin embargo, ésta adquiere un carácter situado, ya que como se mostrará en el apartado de las relaciones cercanas existen condiciones en otras esferas en las que estas expresiones están restringidas. 
Por otra parte, en relación con la utilidad que dan las mujeres a los ingresos económicos que ellas generan, Chablé et al. (2007) consideran que al ser destinados para los hijos ("el ser para otros") no es una acción que las empodere; sin embargo, otras investigaciones han señalado que la maternidad en contextos violentos o donde las acciones y la libertad de las mujeres son limitadas puede originar experiencias de gratificación, un sentido de autoridad, de control y poder de toma de decisiones, que les permite afirmarse y fortalecerse (Ortiz-Ortega et al., 2006; Petchesky, 2006; Seeman, 2003).

\section{Dimensión colectiva del EMPODERAMiento}

En este apartado se identificó que las condiciones que potenciaron la organización colectiva y sostenimiento del grupo de mujeres entrevistadas fueron el reconocimiento de las habilidades de cada una de sus compañeras para abrir espacios y reunirse, y para responder colectivamente frente a dificultades personales y en sus actividades como grupo, además del uso de tiempo para la recreación.

Nosotras donde ya nos organizamos fue donde, qué te gusta a ti, por decir a Blanca, dice 'a mí me gusta bordar' y ya entonces ella borda y borda muy bonito, y Bertha dice 'yo quiero pintar' y pinta y cose, y entonces a mí me gusta mucho dibujar y pintar y bordar también, pero eso se le da mejor a Blanca (Susana, producción de nopal y crianza de cerdos).

Los criábamos chiquitos (a los cerdos), entonces ya les lavábamos el chiquero, ya las señoras nos dividíamos entre dos para ir a hacerles el aseo, teníamos cuatro marraneras[...] entonces nos tocaba de dos en dos para ir a lavarles y echarles de comer en la mañana y en la tarde, y ya otro día otras dos hasta que ya los criamos (Blanca, producción de nopal y crianza de cerdos).

Por decir, que yo necesitaba y a mí me prestaban lo que yo necesitaba, nomás que se firmaba un papel, y ya de ahí haga de cuenta que yo lo tenía que pagar en el tiempo que me decía, y así pues nos prestábamos el dinero y nos ayudábamos mucho (Bertha, producción de nopal y crianza de cerdos).

El reconocimiento de las motivaciones y capacidades de cada una de las participantes, como se aprecia, fue de suma importancia en sus procesos de organización para el trabajo en conjunto; también lo fue la asignación equitativa de actividades y el apoyo mutuo.

Otros aspectos relevantes que se observaron para su sostenimiento como grupo fueron sus espacios y tiempos para la recreación.

También hay que echar relajo, es que siempre en esas horas, como nosotras cuatro ya nos avenimos bien, ya en todo salimos de acuerdo, entonces pues uno siempre se acopla a las otras y por eso a lo mejor hemos durado (Blanca, producción de nopal y crianza de cerdos).

Contrario a lo señalado hasta el momento en relación con los elementos que facilitaron la organización y sostenimiento como grupo, otras mujeres que no participan en este grupo expresaron su inquietud de reunirse con otras para desarrollar proyectos en conjunto, pero indicaron las dificultades que han enfrentado para lograrlo.

Yo les comparto lo que sé a otras mujeres de la comunidad, procuro decirles de las plantas, dónde crecen, para qué sirven[...] Me fijo primero en su necesidad y luego en la manera de ser de las personas, no negativas, yo no quiero que me cuentes de tu vecina ni de tu hermano, ni de nadie, yo no quiero saber nada, no defectos ni detalles de nada, aquí vamos a trabajar y esto te va a servir para olvidarte de los problemas[...] lo negativo no me hace bien, no te deja nada bueno[...] En estas comunidades pequeñas es lo que más importancia le da la gente (Sonia, productos de higiene personal herbolarios).

El chisme sí existe, por ejemplo, a mí me traen de ratera[...] me tachan de ratera porque soy la presidenta del comité del agua, entonces claro que las personas que dicen que yo me robé o que soy ratera es porque realmente no estuvieron al pendiente desde que se hacían las juntas, donde una les da a conocer qué pasó, entonces de ahí se hace un mal comentario, entonces es pues gente negativa (Gracia, huertos de traspatio). 
Como se puede notar, de la primera cita la participante muestra motivación y una actitud de apertura para trabajar con otras mujeres, condiciones que abonan a la configuración del trabajo en grupo; sin embargo, en los dos casos, el chisme en palabras de Vázquez (2008) aparece como un mecanismo de contraempoderamiento en las participantes, es decir, como un dispositivo que intenta minar sus esfuerzos de autonomía, de independencia y control de sus propias vidas, así como una barrera psicosocial para la organización colectiva.

Otros elementos identificados que no contribuyeron a la organización colectiva de las mujeres para participar en proyectos fueron la falta de tiempo y el incremento de trabajo que representa para participar en ellos.

Primero trabajé en una fábrica como dos años, pero se me hacía mucho el trabajo el de aquí de la casa y el de allá, y pues con el proyecto sería lo mismo (Fernanda, huertos de traspatio).

Yo le voy a decir una cosa, yo a mis hijos los tengo muy mal acostumbrados y a mi esposo también, en lo de la casa que barrer, que los trastes, ellos no, ellos pues se van a ver a sus animales (Irene, huertos de traspatio).

Como se puede advertir, una limitación importante para que las mujeres participen en proyectos grupales es la carga de trabajo doméstico y su distribución inequitativa entre los miembros de la familia. Este elemento que no facilita el empoderamiento en la dimensión colectiva tampoco contribuye a la transformación de la estructura de género en la dimensión de las relaciones cercanas e impacta en la dimensión individual.

\section{Dimensión de las relaciones Cercanas}

En esta esfera y a partir de las actividades que desempeñan las participantes para el sostenimiento de la vida familiar: domésticas, de cuidado y de generación de ingresos económicos, se evidencia que su tránsito en las esferas privada y pública no se da de forma antagónica sino complementaria. Esto no se traduce en un mejoramiento de sus condiciones de vida y, por el contrario, les implica una doble o triple jornada laboral, lo cual está vinculado con el carácter relacional del género y con los márgenes de acción del contexto para actuar, es decir, el empoderamiento en esta dimensión no sólo comprende cambios en los comportamientos y expectativas de las mujeres, sino también transformaciones en las expectativas y conductas de sus parejas y familia cercana.

Es que sí se le complica a una[...] Nosotras las mujeres en las tardes los limpiamos (los nopales), en los ratos que tenemos libre[...] decimos, no pues, vamos a la una para que cuando vengan los señores a comer ya andamos de vuelta, y ya nos regresamos a casa a hacer el trabajo que nos queda (Blanca, producción de nopal y crianza de cerdos).

Aquí en la casa yo hago todo y él nada, a él ya se le acabó la obligación y a nosotros como mujeres nunca se nos acaba (Fernanda, huertos de traspatio).

En mí caso, como ahorita que ya terminé, ya ahorita les hago la comida y cuando llegan ya cenan y ya, pero así que mi marido y mis hijos hagan quehacer, no (Lourdes, huertos de traspatio).

Como se comentó, la distribución inequitativa del trabajo en casa es un indicador de una falta de condiciones ideológicas para la transformación de las relaciones de género; el sostenimiento de la vida familiar a través de las labores domésticas, de crianza y de cuidado sigue siendo un tema de las mujeres aun cuando también contribuyan de forma remunerada mediante su participación en actividades que les generan ingresos económicos. De esta forma, se identifica que la escasa o nula participación de los hombres en las actividades del hogar es una condición que no facilita el empoderamiento de las mujeres en las dimensiones individual, colectiva y en las relaciones cercanas.

Yo tengo que pedirle permiso a mi esposo para ir a alguna parte, yo no me mando sola[...] A mí también se me hace buen ejemplo para mis hijos[...] en ese aspecto de que también valoren a su pareja, le den su lugar[...] para que dure (Sonia, huertos de traspatio). 
Toda la vida he trabajado, cuando me casé él no me dejó trabajar, y el error de las mamás, si el marido dice celeste, aunque sea rosa, pues es celeste (Paula, huertos de traspatio).

La falta de autonomía y de toma de decisiones instrumentales al interior de la unidad doméstica sugiere que no existen cambios estructurales en las relaciones de género. Al respecto, se observa que las normas socioculturales de género vinculadas con el funcionamiento familiar expresadas en roles y estereotipos en orden a preservar valores tradicionales de ser buenas madres y esposas que las participantes asumen y reproducen tienen un peso sustantivo y restrictivo en sus procesos de empoderamiento, refuerzan las desigualdades de género y constituyen un riesgo para la justificación y legitimación de la violencia de género.

\section{DisCUSIÓN Y CONCLUSIONES}

El propósito del artículo fue conocer a través de las experiencias concretas de las mujeres rurales del sureste de Coahuila si su participación en proyectos productivos tenía un impacto o no en sus procesos de empoderamiento en las dimensiones individual, colectiva y de las relaciones cercanas. Al respecto, se aprecia que si bien los financiamientos que recibieron las mujeres con la intención de empoderarlas económicamente abonan de forma escasa y complementaria a otras fuentes de subsistencia familiar y contribuyen al autorreconocimiento de algunas de sus capacidades psicosociales, incrementan su carga de trabajo sin tener un peso sustantivo en la transformación de sus condiciones de vida materiales, sociales y en las relaciones de género.

No obstante lo anterior, se reconoce que la investigación constituyó una aproximación que orienta que los resultados adquieran un carácter exploratorio y no concluyente.

Un aspecto considerado inicialmente en el diseño de este estudio fue entrevistar a las parejas de las participantes, así como a sus hijas e hijos con el fin de analizar el carácter relacional del género en las dimensiones del empoderamiento citadas; sin embargo, debido a las dificultades en sus tiempos y el acceso a ellos, no fue posible recolectar esa información.

Otro tema importante, en tanto factor que inhibe los procesos de empoderamiento y que no se abordó con mesura en este trabajo, fue la violencia de género. Al respecto, existieron relatos en algunas mujeres donde mencionaron ese aspecto a la par que la vergüenza por encontrarse en esa situación, pero al mismo tiempo evitaron hablar del tema. Frente a esto, se reconoce la necesidad de desarrollar dispositivos metodológicos para explorar y comprender cómo las normas culturales articuladas con desigualdades de clase afectan el grado y las características de la violencia en el sector rural, esto como eje de análisis fundamental en el estudio de los procesos de empoderamiento.

Por otra parte, son varios los puntos de reflexión que deja la experiencia en este trabajo. Metodológicamente un punto que abre la discusión sobre la concepción de las mujeres rurales y las estrategias utilizadas para potenciar su empoderamiento parte de la lectura que se da a lo difícil que resultó el acercamiento e identificación con las sujetas sociales en la zona de estudio, lo cual está vinculado con la demora en el apoyo solicitado a las instancias de gobierno para identificar y contactar a las participantes, una hipótesis parte del siguiente planteamiento.

De acuerdo con Rubio (2009), el impulso de políticas neoliberales en nuestro país condujo a que un grupo reducido de empresas agroalimentarias transnacionales controlara todos los aspectos de la vida agropecuaria y que la producción de los pequeños y medianos productores quedara en el lugar de autoconsumo o bien abandonaran o rentaran la tierra y migraran en busca de mejores condiciones de vida. Esto se refleja en información proporcionada por Subsidios al Campo en México (2018), donde se menciona que durante el gobierno del sexenio 2006-2012 las grandes empresas (un 20\% del padrón) recibieron 60\% de los subsidios, mientras que 40\% restante se repartió entre los pequeños y medianos productores que conformaron $80 \%$ del padrón de los beneficiados por la Secretaría de Agricultura y Desarrollo Rural (SAGARPA) durante ese periodo. Asimismo, de acuerdo con la misma instancia, la pequeña agricultura representa 39\% de la producción agrícola nacional y $73 \%$ lo constituyen los pequeños productores. 
Esta situación, aunada al panorama económico de Coahuila, donde 10\% de la población es rural (Censo de Población y Vivienda, 2010) y las actividades primarias aportan sólo 3\% al Producto Interno Bruto (PIB) estatal en comparación con las actividades secundarias (52\%) y las actividades terciarias (45\%) (Instituto Nacional de Estadística e Informática, 2014), lleva a apreciar que existe una falta de atención en el sector rural, específicamente en los pequeños productores o campesinos quienes no participan en las actividades agroindustriales o de las grandes empresas que abonan al PIB del estado.

Esta condición advierte que el acercamiento con las sujetas de estudio y el contexto demanda una vinculación que permita concebir de forma distinta a la que se realiza desde el gabinete. Existe la apreciación que desde este ámbito se les visualiza sin agencia y carentes de poder y, por tanto, se justifica la propuesta de "empoderarlas" a través de programas sociales basados en enfoques antipobreza, los cuales, como se observó en esta investigación, si bien contribuyen a potenciar recursos personales psicosociales en la esfera individual y favorecen en alguna medida el bienestar de sus hijas e hijos, no transforman sus condiciones de vida materiales y sociales.

Tal como lo muestran los resultados, es una realidad que las mujeres pobres y en condiciones de ruralidad resisten a las circunstancias injustas e indignas en que viven. Es por ello que se reconoce la importancia de centrarse en la perspectiva del punto de vista de estas mujeres con el objetivo de evidenciar las distintas estrategias que utilizan individual y colectivamente para enfrentar las demandas situacionales económicas y sociales en sus vidas, así como sus contribuciones en el sostenimiento de la vida y reproducción social. Empero, tenemos que cuidar no caer en concepciones voluntaristas que consideren que acciones individuales pueden subsanar condiciones estructurales privativas.

Otro punto de discusión y cuestionamiento es la separación de las esferas pública y privada. Como se expuso, las participantes, además de desempeñar labores domésticas, de crianza y de cuidado, desarrollan actividades instrumentales tanto en la dimensión privada (huertos de traspatio, cría de animales, elaboración de productos medicinales y de higiene personal herbolarios), como en la esfera pública (venta de tales productos, así como alimentos procesados, o no, en oficinas gubernamentales y mercados municipales) para asegurar la supervivencia de sus familias. Sin embargo, los sesgos androcéntricos inscritos en la economía capitalista ven a estas actividades como complementarias y carentes de valor económico y social.

Por lo descrito, no obstante que se reconoce la importancia de no perder una mirada crítica respecto al papel de Estado y los efectos del modelo económico prevaleciente en las formas de vida y subjetividades de las personas, una epistemología feminista que permita redirigir miradas dominantes basadas en sesgos androcéntricos y capitalistas es un pilar fundamental para hacer visibles las expresiones de agencia de las mujeres, sus estrategias de vida y de resistencia y las contribuciones de las actividades que realizan para el sostenimiento de la vida familiar.

Del mismo modo, también es significativa para repensar los marcos teóricos y de análisis que permitan el reconocimiento de otras economías, por ejemplo, de una economía del cuidado que, en palabras de Carrasco (2012), abone a la satisfacción de las necesidades de todos los seres humanos manteniendo la capacidad de reproducción de su mundo común social y natural, y ponga a la producción y al mercado al servicio de las comunidades y las personas.

\section{Prospectiva}

El empoderamiento es un proceso situado que requiere de políticas distributivas justas de los recursos y la riqueza, así como de políticas de reconocimiento de las perspectivas características de las mujeres rurales pobres (Fraser, 2008), las cuales en su integración permitan facilitar condiciones objetivas y subjetivas que amplíen los márgenes de acción y potencien las capacidades de las personas.

Además, se sugiere realizar esfuerzos y utilizar metodologías críticas, alternativas y contextualizadas que permitan comprender cómo las condiciones materiales enmarcan los referentes existenciales de las mujeres rurales y, atendiendo su propia perspectiva, que sean ellas las protagonistas del proceso de construcción de conocimiento, así como en la detección de problemas y necesidades y en la elaboración de propuestas y soluciones. 
Para concluir, se identifican como líneas de investigación significativas futuras en el abordaje de los procesos de empoderamiento en las mujeres rurales las siguientes: $a$ ) una economía del cuidado que, desde una epistemología feminista, haga visible las aportaciones del trabajo no remunerado de las mujeres en las unidades de producción campesina y en el sostenimiento de la vida familiar. b) Profundizar en el estudio de la violencia a fin de identificar cómo las normas culturales articuladas con desigualdades económicas en el contexto rural afectan el grado y las características de la violencia que sufren las mujeres. c) El estudio del chisme como una barrera psicosocial que no abona al empoderamiento colectivo de las mujeres, así como su vínculo con la violencia de género. $d$ ) Con base en lo referido por las mujeres, particularmente de San José de la Joya, analizar y comprender el impacto que tiene la escasez del agua en sus dinámicas de vida, así como en sus patrones de conducta, pero también conocer de qué forma participan en su gestión y cuál es su impacto en sus procesos de empoderamiento.

\section{Agradecimientos}

Se agradecen los comentarios de los árbitros de la revista que mejoraron sustancialmente el contenido del artículo.

\section{REFERENCIAS}

Aguilar Pinto, E. C., Tuñón Pablos, E., Zapata Martelo, E. y Evangelista García. A. (2017). Mujeres, empoderamiento y microcrédito. El programa de microempresas sociales de Banmujer en Chiapas. Agricultura, Sociedad y Desarrollo, 14(3), 479-501.

Alberti P., Zavala H., M., Salcido R., B. y Real L., N. (2014). Género, economía del cuidado y pago del trabajo doméstico rural en Jilotepec, Estado de México. Agricultura, Sociedad y Desarrollo, 11 (30), 379-400.

Banco Mundial (Banco Internacional de Reconstrucción y Fomento/Banco Mundial). (2011). Informe sobre el Desarrollo Mundial 2012. Igualdad de Género y Desarrollo. Panorama General. Washington, D. C. Disponible en http://siteresources.worldbank.org/INTWDR2012/Resources/7778105-1299699968583/7786210-1315936231894/Overview-Spanish.pdf

Barreda, T. L. D. y Gándara, F., G. (2007). Empoderamiento femenino y desarrollo rural: evaluación de un programa de desarrollo regional sustentable en Cuatro Ciénegas, Coahuila. Documento de Trabajo No.2007-4. Cátedra de Integración Económica y Desarrollo Social. Tecnológico de Monterrey.

Bartra, E. (2012). Acerca de la investigación y metodología feminista, en G. N. Blazquez; P. F. Flores y E. Ríos (Coords). Investigación feminista, metodología y representaciones sociales. México: UNAM, CEIICH, CRIM.

Batliwala, S. (1997). El significado del empoderamiento de las mujeres: nuevos conceptos desde la acción, en M. León (Comp.), Podery Empoderamiento de las mujeres (pp. 187-211). Santa Fé de Bogotá: Tercer Mundo.

Braidotti, R. (2004). Mujeres, medio ambiente y desarrollo sustentable. Surgimiento del tema y diversas aproximaciones, en G. Vázquez, V. y M. Velázquez G. (Comps.), Miradas al Futuro. Hacia la construcción de sociedades sustentables con equidad de género (pp. 23-59). México: UNAM/Colegio de Posgraduados/ Centro Internacional de Investigaciones para el Desarrollo.

Carrasco, C. (2012).Economía, trabajos y sostenibilidad de la vida. Sostenibilidad de la vida. Aportaciones desde la Economía Solidaria, Feminista y Ecológica. Bilbao: Universidad del País Vasco. 
Carrasco, B. C. (2006). La economía feminista: una apuesta por otra economía, en M. M. J. Vara (Coord.), Estudios sobre género y economía (pp. 29-62). España: Akal.

Carr, M. (2002). El empoderamiento económico de las mujeres: clave de desarrollo, en L. de Pauli (Ed.), Mujeres: empoderamiento y justicia económica. Reflexiones de la experiencia en Latinoamérica y el Caribe. México: Fondo de Desarrollo de las Naciones Unidas para la Mujer.

Chablé, E., Gurri, G. F., Molina R., D. y Schmook, B. (2007). Fuentes de ingreso y empoderamiento de las mujeres campesinas en el municipio de Calakmul, Campeche. Politica y Cultura, 28, 71-95.

CONEVAL (Consejo Nacional de Evaluación de la Política de Desarrollo Social). (2016). Informe de Pobreza en México. Disponible en https://www.coneval.org.mx/Medicion/MP/Paginas/Pobreza_2016.aspx

CONEVAL (Consejo Nacional de Evaluación de la Política de Desarrollo Social). (2015). Informe de Evaluación de la Politica de Desarrollo Social en México 2014. D. F. Disponible en https://www.coneval.org. $\mathrm{mx} /$ Informes/Evaluacion/IEPDS_2014/IEPDS_2014.pdf

Creswell, J. W. (1998). Qualitative inquiry and research design. Choosing among five traditions. Thousand Oaks, CA: Sage.

Deere, D. y León, M. (2002). Género, propiedad y empoderamiento. Tierra, Estado y Mercado en América Latina. México: UNAM.

FAO (Food and Agriculture Organization). (2006). Agricultura, expansión del comercio y equidad de género. Roma. Disponible en http://www.fao.org/docrep/009/a0493s/a0493s03.htm\#bm3

Fraser, N. (2008). La justicia social en la era de la política de la identidad: redistribución, reconocimiento y participación. Revista de Trabajo, 4(6), (83-99).

Ghebre-Medhin, T. y Restrepo, E. (2002). Microfinanciamiento plus: una iniciativa conjunta en América Latina, en L. de Pauli (Ed.). Mujeres: empoderamiento y justicia económica. Reflexiones de la experiencia en Latinoamérica y el Caribe (pp. 115-130). México: Fondo de Desarrollo de las Naciones Unidas para la Mujer.

González, S. y Rosas V., R. (2013). Los proyectos productivos de las mujeres rurales como una alternativa para eliminar la pobreza. International Journal of Latin American Studies, 3(1), 33-63.

Gutiérrez, V., Nazar B., A., Zapata M., E., Contreras U. J., y Salvatierra I., B. (2013). Género y participación de las mujeres en la gestión del agua en las subcuencas Río Sabinal y Cañón del Sumidero, Berriozábal, Chiapas. La ventana, 4(38), 246-275.

Hidalgo N. (2002). Género, empoderamiento y microfinanzas. Un estudio de caso en el norte de México. México: Instituto Nacional de las Mujeres.

INEGI (Instituto Nacional de Estadística e Informática). (2014). Sistema de Cuentas Nacionales de México 2008. Participación por actividad económica, en valores corrientes, 2014. Cifras preliminares.

INEGI (Instituto Nacional de Estadística e Informática). Censo de Población y Vivienda 2010.

Kabeer, N. (1997). Empoderamiento desde abajo: ¿qué podemos aprender de las organizaciones de base?, en León (Comp.), Poder y empoderamiento de las mujeres (pp. 119-146). Santa Fé de Bogotá: Tercer Mundo.

Kvale, S. (2007). Doing interviews. Thousand Oaks, CA: Sage Publications.

Lagarde, M. (2012). El feminismo en mi vida. Hitos, clavesy topías. México: Gobierno de la Ciudad de México, Instituto de las Mujeres de la Ciudad de México.

Lagarde, M. (1996). Género y feminismo. Desarrollo bumano y democracia. España: Horas y Horas.

León, M. (2003). Poder y empoderamiento de las Mujeres. Bogotá. 
León, M. (1997). El empoderamiento en la teoría y práctica del feminismo, en M. León (Comp.), Poder y empoderamiento de las mujeres (pp. 1-26). Santa Fé de Bogotá: Tercer Mundo.

López, V., y Rojas L., V. (2017). Rezagos en el nivel de autonomía de las mujeres rurales mexicanas en la primera década del siglo XXI. Estudios Demográficos y Urbanos, 32(2), 315-354.

Martínez L., E., Zapata M., E., Alberti M., P. y Díaz C., R. (2005). Género y poder en tres organizaciones rurales de la región Lagunera. Revista Mexicana de Sociología, 67(2), 271-319.

Mingo, A. (1996). El sinuoso camino de las organizaciones productivas de campesinas. Estudios Sociológicos, 14(40), 75-95.

Mora, M., Fernández D., M. C. y Ortega O. S., V. (2016). Asociacionismo productivo y empoderamiento de mujeres rurales. Madres multiactivas, socias y mujeres campesinas. CUHSO. Cultura-Hombre-Sociedad, 26(1), 131-160.

Moser, C. (1991). Planificación de género y desarrollo. Teoria, práctica y capacitación. Lima: Red entre Mujeres/ Flora Tristán.

Ortiz-Ortega, A., Amuchástegui, A. y Rivas., M. (2006). Porque yo los traje al mundo. La negociación de los derechos de las mujeres en México, en P. R. Petchesky y K. Judd (Comps.), Cómo negocian las mujeres sus derechos en el mundo. Una intersección entre culturas, politica y religiones (pp. 251-300). México: Colegio de México

Pérez, A., Vázquez G., V. y Zapata M., E. (2008). Empoderamiento de las mujeres indígenas de Tabasco. El papel de los Fondos Regionales de CDI. Cuicuilco, 15(42), 165-179.

Patton, M. (1990). Qualitative evaluation and research methods. Beverly Hills, CA: Sage.

Petchesky P., R. (2006). Introducción, en P. R. Petchesky y K. Judd (Comps.), Cómo negocian las mujeres sus derechos en el mundo. Una intersección entre culturas, politica y religiones (pp. 39-83). México: Colegio de México.

Riaño, E. y Okali, C. (2008). Empoderamiento de las mujeres a través de su participación en proyectos productivos: experiencias no exitosas. Convergencia. Revista de Ciencias Sociales, 46, 119-141.

Rowlands, J. (1997). Empoderamiento y mujeres rurales en Honduras: un Modelo para el Desarrollo, en M. León (Comp.), Poder y empoderamiento de las mujeres (213-245). Santa Fé de Bogotá: Tercer Mundo.

Rubio, B. (2009). El impacto de la crisis alimentario en las mujeres rurales de bajos ingresos en México 2008-2009. México. Cámara de Diputados LX Legislatura. Red Nacional de Promotoras y Asesoras Rurales. INDESOL. Disponible en http://biblioteca.diputados.gob.mx/janium/bv/ce/scpd/LX/impac_cris_muj.pdf

Ruiz, M. L.E. (2012). Relaciones de género y mercados de derechos de agua y tierra en Chiapas. Región y sociedad, 24(53), 55-89.

Seeman, I. (2003). Subjetion, subjectivity and agency: The meaning and practice of mothering within the context of domestic violence. Paper presented at the Annual Meeting o the American Sociological Association. Atlanta GA.

Subsidios al Campo en México. (2018). Campo mexicano. Un retrato de desigualdad, explotación e impunidad Disponible en http://subsidiosalcampo.org.mx/nota/campo-mexicano-un-retrato-de-desigualdad-explotacion-e-impunidad/

Tuñon Pablos, E. (2011). Evaluación de los programas de créditos a proyectos productivos de mujeres en Tabasco, Campeche y Quintana Roo. La Ventana, 32, 81-116.

Vázquez, V. (2008). El Chisme y la violencia de género. En búsqueda de vínculos, en R. Castro e I. Casique (Ed.), Estudios sobre cultura, género y violencia contra las mujeres (pp.139-172). México: UNAM. 
Vázquez-Luna, D., Mortera P., D., Rodríguez-Orozco, N., Martínez M., M. y Velázquez S., M. G. (2013). Organización comunitaria de mujeres: del empoderamiento al éxito del desarrollo rural sustentable. La Ventana, $37,262-288$.

Young, K. (1997). El potencial transformador en las necesidades prácticas: empoderamiento colectivo y el proceso de planificación, en León (Comp.) Poder y empoderamiento de las mujeres (pp.147-172). Santa Fé de Bogotá: Tercer Mundo.

Notas

[1] Las dimensiones de pobreza según el CONEVAL están relacionadas con la carencia de bienestar económico (ingreso) y la falta de acceso a derechos sociales que traen como consecuencia: rezago educativo, falta de acceso a la educación, a los servicios de salud, a la seguridad social, a la alimentación, a los servicios básicos de vivienda y a la falta de calidad y servicios de vivienda.

[2] El propósito de este instrumento estadístico desarrollado y aplicado por el Instituto Nacional de Estadística y Geografía tiene como objetivo conocer el valor económico del trabajo no remunerado que los miembros del hogar realizan en actividades productivas para la generación de servicios destinados a la satisfacción de sus necesidades, así como su aporte a la economía nacional.

[3] El empoderamiento económico de acuerdo con Carr (2002) se define como el hecho de tener acceso y control de los medios necesarios para poder vivir en condiciones sostenibles a largo plazo y de recibir los beneficios de dicho acceso y control.

[4] Los nombres referidos de las mujeres entrevistadas son pseudónimos para cumplir con los criterios éticos de anonimato y confidencialidad.

\section{BY-NC-ND}

